\title{
Statyba
}

\section{ROLE OF KUHN-TUCKER CONDITIONS IN ELASTICITY EQUATIONS IN TERMS OF STRESSES}

\section{E. Chraptovič \&J. Atkočiūnas}

To cite this article: E. Chraptovič \& J. Atkočiūnas (2000) ROLE OF KUHN-TUCKER CONDITIONS IN ELASTICITY EQUATIONS IN TERMS OF STRESSES, Statyba, 6:2, 104-112, DOI: $10.1080 / 13921525.2000 .10531573$

To link to this article: https://doi.org/10.1080/13921525.2000.10531573

曲 Published online: 26 Jul 2012.

Submit your article to this journal $\pi$

III Article views: 55 


\title{
РОЛЬ УСЛОВИЙ КУНА-ТАККЕРА В ФОРМУЛИРОВКЕ УРАВНЕНИЙ ТЕОРИИ УПРУГОСТИ В НАПРЯЖЕНИЯХ
}

\author{
Э. Храптович, Ю. Аткочюнас \\ Вильнюсский технический университет им. Гедиминаса
}

\section{1. Введение}

Решение задачи теории упругости в напряжениях связано с определением шести компонентов вектора напряжений из девяти уравнений (равновесия и неразрывности) [1-3]. Непосредственное интегрирование системы девяти дифференциальных уравнений в частных производных относительно шести неизвестных функций напряжений затруднительно. Поэтому для решения краевой задачи теории упругости в напряжениях применяется вариационный метод Кастильяно [4-6]. В середине XX века Саусвеллом и Кпошниковым [4, 7] было доказано, что применение метода Кастильяно приводит к автоматическому выполнению всех шести уравнений совместности деформаций. Однако вопрос о числе независимых уравнений совместности при этом не исследовался. Оставалось неясным, содержит ли постановка задачи теории упругости в напряжениях переопределенную систему уравнений. Только в работах $[5,8]$ и других с использованием вариационного уравнения Кастильяно показано, что независимых уравнений совместности деформаций всего три, а не шесть (при преобразованном виде краевых условий). Однако исчерпывающим образом уравнения метода сил (уравнения совместности в напряжениях) в упомянутых работах не исследовались.

В настоящей работе вопрос о числе независимых уравнений совместности деформаций напрямую не рассматривается. Авторами для построения математической модели задачи теории упругости в напряжениях (уравнений метода сил) используется теория математического программирования в функциональном пространстве. На основе принципа Кастильяно (принципа минимума дополнительной энергии) строится экстремальная задача для определения действительных напряжений $[3,9,10]$. Показано, что условия
Куна-Таккера $[10,11]$ для оптимального решения этой задачи и являются уравнениями совместности деформаций упругого тела (конкретный вид условий Куна-Таккера зависит от формы зависимостей, определяющих статически возможный вектор напряжений). В отличие от работ других авторов здесь статически возможный вектор строится с привлечением функций напряжений $\varphi$. В настоящем исследовании для доказательств и рассуждений не привлекалась задача математического программирования, двойственная к исходной экстремальной задаче.

Оговорим принципы изложения материала в представляемой работе. Во втором разделе рассматриваются условия Куна-Таккера для задачи математического программирования при наличии ограничений в качестве как равенств, так и неравенств. В третьем разделе приводится математическая модель задачи определения действительного напряженного состояния упругого тела, зачастую называемая задачей анализа. Разработке методики применения условий Куна-Таккера отводятся четвертый (плоская задача теории упругости) и пятый (трехмерная задача) разделы.

\section{2. Условия Куна-Таккера в задачах}

\section{математического программирования}

Рассматривается задача минимизации некоторой функции при наличии ограничений в форме как равенств, так и неравенств:

найти

$$
\min \bar{\pi}(\mathbf{x})
$$

при условиях

$$
\begin{aligned}
& g_{i}(\mathbf{x}) \leq 0, \quad i=1,2, \ldots, m \\
& h_{i}(\mathbf{x})=0, \quad i=1,2, \ldots, l .
\end{aligned}
$$


Условия Куна-Таккера $[10,11]$ связаны с оптимальным решением исходной задачи $\mathbf{x}^{*}$ (1)-(3). Для выпуклых функций условия Куна-Таккера являются условиями глобального решения исходной задачи и гласят: $\mathbf{x}^{*}$ является оптимальным решением исходной задачи, если можно найти такие числа $\lambda_{i}(i=1,2, \ldots, m), u_{i}(i=1,2, \ldots, l)$, при которых

$$
\begin{gathered}
\nabla \mathscr{F}\left(\mathbf{x}^{*}\right)+\nabla \mathbf{g}^{T}\left(\mathbf{x}^{*}\right) \boldsymbol{\lambda}+\nabla \mathbf{h}^{T}\left(\mathbf{x}^{*}\right) \mathbf{u}=\mathbf{0} \\
\lambda^{T} \mathbf{g}\left(\mathbf{x}^{*}\right)=0 \\
\lambda \geq \mathbf{0}
\end{gathered}
$$

Зависимость (4) обозначает, что для оптимального решения задачи (1)-(3) градиент функции цели $\nabla \mathscr{F}\left(\mathbf{x}^{*}\right)$ является линейной комбинацией градиентов ограничений (см. рис. для двухмерной задачи):

$$
\nabla \mathscr{F}\left(\mathbf{x}^{*}\right)=-\sum_{i=1}^{m} \nabla \mathrm{g}_{i}\left(\mathbf{x}^{*}\right) \lambda_{i}-\sum_{i=1}^{l} \nabla \mathrm{h}_{i}\left(\mathbf{x}^{*}\right) u_{i}
$$

Составляющие векторов $\lambda$ и $\mathbf{u}$ являются множителями Лагранжа, а равенства $\lambda_{i} \mathrm{~g}_{i}\left(\mathbf{x}^{*}\right)=0, \lambda_{i} \geq 0$, $i=1,2, \ldots, m-$ условиями о дополняющей нежесткости.

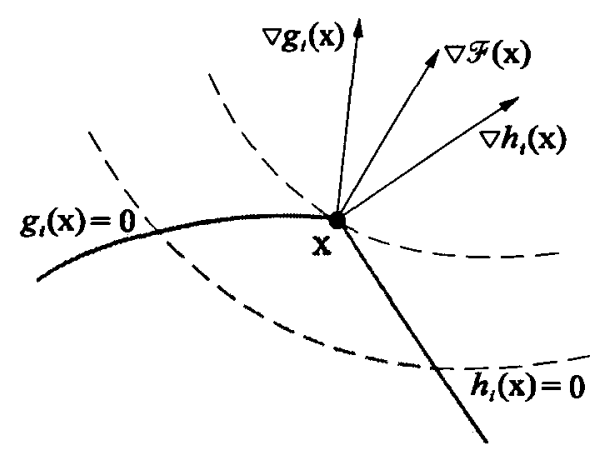

К иллюстрации условий Куна-Таккера

Illustration for Kuhn-Tucker conditions

Если оптимальное решение исходной задачи $\mathbf{x}^{*}$ неизвестно, его можно определить из системы, состоящей из условий Куна-Таккера (4)-(6) и ограничений (2)-(3) исходной задачи (1)-(3):

$$
\begin{aligned}
& \mathbf{g}(\mathbf{x}) \leq \mathbf{0}, \\
& \mathbf{h}(\mathbf{x})=\mathbf{0} \\
& \nabla \mathscr{H}(\mathbf{x})+\nabla \mathbf{g}^{T}(\mathbf{x}) \lambda+\nabla \mathbf{h}^{T}(\mathbf{x}) \mathbf{u}=\mathbf{0} \\
& \lambda^{T} \mathbf{g}(\mathbf{x})=0, \\
& \lambda \geq \mathbf{0} .
\end{aligned}
$$

В теории упругости (пластичности) зависимости (7) называются полной системой уравнений теории упругости или уравнениями Эйлера-Лагранжа.

\section{3. Экстремальная задача определения действительных напряжений}

В рамках допущений линейной теории упругости рассматривается ограниченное поверхностью $A$ тело объемом $V$. Соотношения геометрически линейной механики основаны на предположении о малости перемешений по сравнению с линейными размерами деформируемого тела. На части поверхности такого идеально упругого тела $A_{f}$ задана поверхностная нагрузка $\mathbf{q}$, а на поверхности $A_{u}$ - перемешения $\mathbf{u}=\mathbf{0}$ (принимается, что объемные силы отсутствуют). Деформированное состояние упругого тела характеризуется векторами перемещений $\mathbf{u}=\left\{\mathrm{u}_{1}, \mathrm{u}_{2}, \mathrm{u}_{3}\right\}^{T}$ и деформаций $\varepsilon=\left\{\varepsilon_{11}, \varepsilon_{22}, \varepsilon_{33}, \varepsilon_{12}, \varepsilon_{13}, \varepsilon_{23}\right\}^{T}$, напряженное состояние - шестимерным вектором напряжений $\boldsymbol{\sigma}=\left\{\sigma_{11}, \sigma_{22}, \sigma_{33}, \sigma_{12}, \sigma_{13}, \sigma_{23}\right\}^{T}$. Статически возможные напряжения $\sigma$ удовлетворяют уравнениям равновесия в объеме тела $V$ и статическим граничным условиям на его поверхности $A_{f}$ :

$$
\begin{aligned}
& {[\propto \mathcal{O}] \boldsymbol{\sigma}=\mathbf{0} \in V,} \\
& {[N] \boldsymbol{\sigma}=\mathbf{q} \in A_{f} .}
\end{aligned}
$$

Здесь [£] - днфференпальый оператор уравнений равновесия, $[N]$ - алгебраическая матрица статических граничньх условий. Знак $\epsilon$ указывает на области определения координат, входпших в данное уравнение.

Рассматривается односвязная область. Деформации являются непрерывными функциями наряду со своими первыми и вторыми производными. Поэтому перемещения должны быть непрерывными функциями класса $\mathrm{C}^{3}$ : геометрические зависимости для идеально упругого тела представляются уравнениями

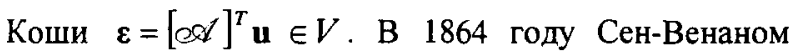
были получены уравнения совместности (сплошности) деформаций для упругого тела - это известная система из шести дифференциальных уравнений [1-3]:

$$
\left.\begin{array}{r}
{\left[\mathscr{A}_{1}\right] \varepsilon_{1}-\left[\mathscr{A}_{2}\right] \varepsilon_{2}=0} \\
-2\left[\mathscr{A}_{4}\right] \varepsilon_{1}+\left[\mathscr{A}_{3}\right] \varepsilon_{2}=0
\end{array}\right\} \text { в объеме }
$$

Здесь $\varepsilon_{1}=\left\{\varepsilon_{11}, \varepsilon_{22}, \varepsilon_{33}\right\}^{T}, \quad \varepsilon_{2}=\left\{\varepsilon_{12}, \varepsilon_{13}, \varepsilon_{23}\right\}^{T}$, 

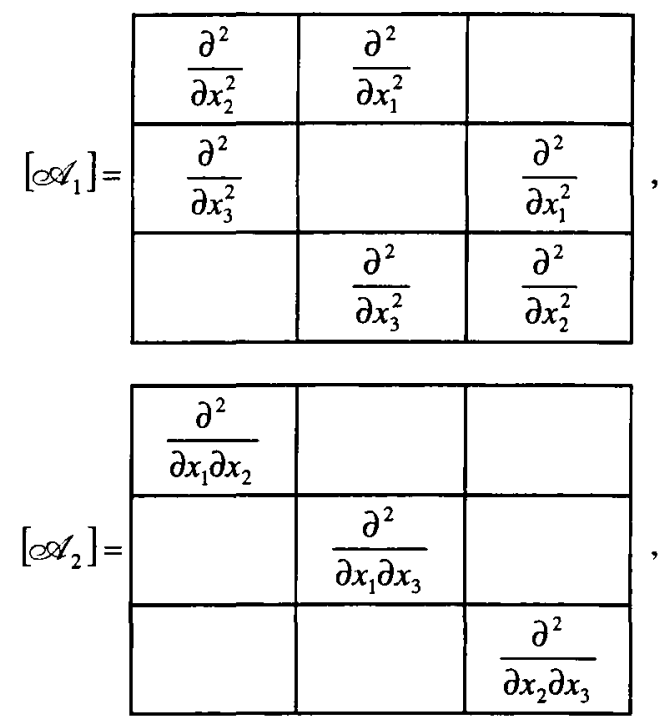

$\left[\mathscr{A}_{3}\right]=$\begin{tabular}{|c|c|c|}
$\frac{\partial^{2}}{\partial x_{1} \partial x_{3}}$ & $\frac{\partial^{2}}{\partial x_{1} \partial x_{2}}$ & $-\frac{\partial^{2}}{\partial x_{1}^{2}}$ \\
\hline$\frac{\partial^{2}}{\partial x_{2} \partial x_{3}}$ & $-\frac{\partial^{2}}{\partial x_{2}^{2}}$ & $\frac{\partial^{2}}{\partial x_{1} \partial x_{2}}$ \\
\hline$-\frac{\partial^{2}}{\partial x_{3}^{2}}$ & $\frac{\partial^{2}}{\partial x_{2} \partial x_{3}}$ & $\frac{\partial^{2}}{\partial x_{1} \partial x_{3}}$ \\
\hline
\end{tabular}

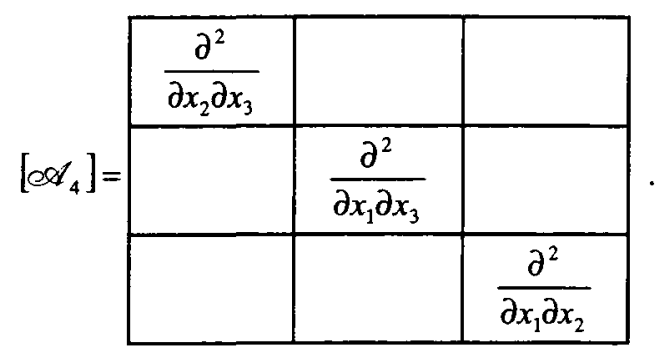

Уравнения (10) можно записать также в виде:

$$
[\mathscr{B}] \varepsilon=0,
$$

где

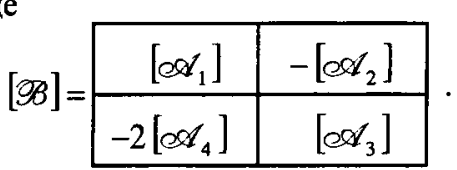

Физический смысл соотношений (10) таков: тело, бывшее сплошным до деформации, остается сплошным после деформации, если удовлетворяются условия (10). Отсюда еще одно название равенств (10) уравнения неразрывности или сплошности деформачий. Следует отметить, что уравнения совместности не налагают никаких ограничений на перемешения, кроме непрерывности их самих и их частных производных. Уравнения неразрывности являются тождествами относительно перемещений. Энергети- ческий смысл заключается в следуюшем: соблюдение неразрывности деформаций соответствует в упругом теле минимальному значению накапливаемой идеально упругим телом потенциальной энергии деформации [2-6]. В несколько иной, но близкой по идее форме, указанный минималистский принцип и его тождественность уравнениям неразрывности деформаций сохраняется и в случае пластических деформаций.

Уравнения Сен-Венана (10) играют решающую роль при построении математической модели краевой задачи теории упругости в напряжениях:

$$
\left.\begin{array}{c}
{[\mathscr{A}] \boldsymbol{\sigma}=\mathbf{0},} \\
{\left[\mathscr{A} \mathscr{A}_{1}\right]\left[D_{1}\right] \boldsymbol{\sigma}_{1}-\left[\mathscr{A _ { 2 }}\right]\left[D_{2}\right] \boldsymbol{\sigma}_{2}=\mathbf{0},} \\
-2\left[\mathscr{A _ { 4 }}\right]\left[D_{1}\right] \boldsymbol{\sigma}_{1}+\left[\mathscr{A _ { 3 }}\right]\left[D_{2}\right] \boldsymbol{\sigma}_{2}=\mathbf{0}
\end{array}\right\} \in V
$$

при граничных условиях

$$
[N] \boldsymbol{\sigma}=\mathbf{q} \text { на } A_{f}, \quad \mathbf{u}=\mathbf{0} \text { на } A_{u} .
$$

Здесь $\left[D_{1}\right],\left[D_{2}\right]$ - подматрицы матрицы податливости $[D]$ обобщенного закона Гука $\varepsilon=[D] \sigma$, $\sigma_{1}=\left\{\sigma_{11}, \sigma_{22}, \sigma_{33}\right\}^{T}, \sigma_{2}=\left\{\sigma_{12}, \sigma_{13}, \sigma_{23}\right\}^{T}$. Шесть уравнений $(116)$ - (11 в) для тела объемом $V$ представляют собой известные уравнения неразрывности в форме Бельтрами [3-5], выведенные им в 1892 году:

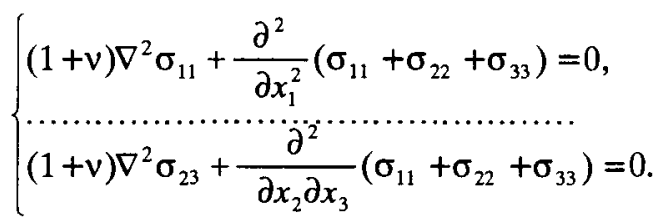

Здесь $\nabla^{2}$ - оператор Лапласа, $v$ - коэффициент Пуассона.

Математическая модель задачи определения действительного напряженного состояния идеально упругого тела строится на основе принципа минимума дополнительной энергии (принцип Кастильяно):

в упругом деформируемом теле из всех статически возможных напряжений действительным напряжениям отвечает минимум дополнительной энергии.

Из этого принципа вытекает следующая экстремальная задача:

найти

$$
\min \mathscr{F}(\sigma)=\frac{1}{2} \int_{V} \sigma^{T}[D] \sigma d V
$$


при условиях

$$
\begin{gathered}
-[\infty \mathcal{d}] \boldsymbol{\sigma}=\mathbf{0} \in V, \\
{[N] \boldsymbol{\sigma}=\mathbf{q} \in A_{f} .}
\end{gathered}
$$

Для идеально упругого тела дополнительная энергия деформирования и потенциальная энергия численно равны между собой. Вследствие положительной определенности матрицы $[D]$ функция क्F $(\sigma)$ представляется положительно определенной квадратичной формой, и задача анализа напряженного состояния тела в статической формулировке (13)-(15) имеет единственное решение $\boldsymbol{\sigma}^{*}$.

Условия Куна-Таккера для задачи анализа (13)-(15) в функциональном пространстве для областей $V$ и $A$ сводятся лишь к зависимостям (4), берущим начало из функции Лагранжа:

$$
\begin{aligned}
& \mathscr{F}_{1}=\frac{1}{2} \int_{V} \boldsymbol{\sigma}^{T}[D] \boldsymbol{\sigma} d V+\int_{V} \mathbf{u}^{T}[\varrho] \boldsymbol{\sigma} d V+ \\
& +\int_{A_{f}} \mathbf{u}^{T}(\mathbf{q}-[N] \boldsymbol{\sigma}) d A .
\end{aligned}
$$

С использованием формулы Гаусса-Остроградского

$$
\int_{V} \mathbf{u}^{T}[\infty d] \boldsymbol{\sigma} d V=-\int_{V} \boldsymbol{\sigma}^{T}[\infty]^{T} \mathbf{u} d V+\int_{A} \boldsymbol{\sigma}^{T}[N]^{T} \mathbf{u} d A \text { (17) }
$$

условия Куна-Таккера для оптимального решения $\sigma^{*}$ задачи (13)-(15) принимают вид:

$$
\begin{aligned}
& {[D] \boldsymbol{\sigma}^{*}-[\mathscr{A}]^{T} \mathbf{u}=\mathbf{0} \in V,} \\
& {[N]^{T} \mathbf{u}=\mathbf{0} \quad \in A_{u} .}
\end{aligned}
$$

Условия (18)-(19) определяют кинематически возможный вектор перемешений. Граничные условия (19) в контексте принятых допущений превращаются в требования $\mathbf{u}=\mathbf{0}$ на $A_{u}$.

Если оптимальное решение задачи анализа (13)-(15) неизвестно, то полная система зависимостей, аналогичная системе (7), для исследуемого случая будет такой:

$$
\left\{\begin{array}{c}
-[\mathscr{A}] \boldsymbol{\sigma}=\mathbf{0}, \\
{[D] \boldsymbol{\sigma}-[\mathscr{Q A}]^{T} \mathbf{u}=\mathbf{0} ;} \\
{[N] \boldsymbol{\sigma}=\mathbf{q} \in A_{f},} \\
\mathbf{u}=\mathbf{0} \in A_{u} .
\end{array}\right\}
$$

Получена полная система уравнений теории упругости.

\section{4. Плоское напряженное состояние}

Вид условий Куна-Таккера зависит также от способа представления зависимостей, определяющих статически возможный вектор напряжений $\sigma$.

Первый способ. Пусть определяющие статически возможный вектор б условия в плоской области $\Omega$ и на ее контуре $\mathscr{L}$ следующие:

уравнения равновесия

$$
-[\infty] \boldsymbol{\sigma}=\mathbf{0} \in \Omega
$$

где

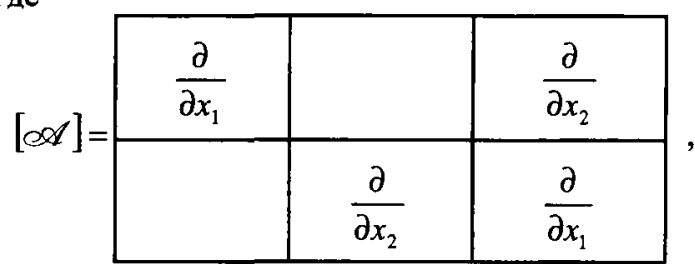

$$
\boldsymbol{\sigma}=\left\{\sigma_{11}, \sigma_{22}, \sigma_{33}\right\}^{T}
$$

статические граничные условия

$$
[N] \sigma=\mathbf{q} \in \mathscr{L}_{f},
$$

где

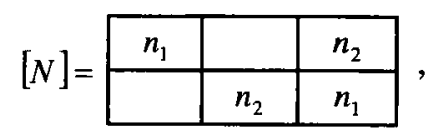

$$
\mathbf{q}=\left\{q_{1}, q_{2}\right\}^{T}
$$

Необходимые и достаточные условия оптимальности Куна-Таккера принимают вид:

$$
\begin{gathered}
{[D] \sigma^{*}-[\mathscr{A}]^{T} \mathbf{u}=\mathbf{0} \in \Omega,} \\
\mathbf{u}=\mathbf{0} \quad \in \mathscr{L}_{\|} .
\end{gathered}
$$

Здесь

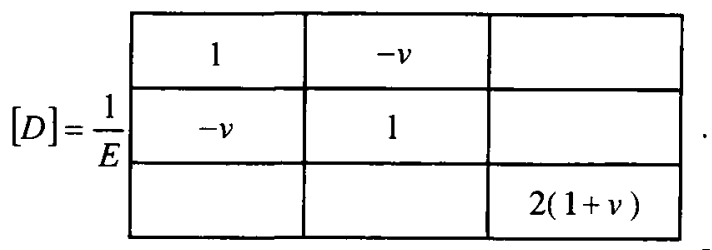

Действительные деформации $\varepsilon^{*}=[D] \sigma^{*}$ $\left(\varepsilon^{*}=\left\{\varepsilon_{11}^{*}, \varepsilon_{22}^{*}, \varepsilon_{12}^{*}\right\}^{\prime}\right)$ входят в геометрические уравнения (25). По теории упругости известно, что однозначно выразить перемещения $\mathbf{u}$ из уравнений 


$$
\boldsymbol{\varepsilon}^{*}-[\mathscr{A}]^{T} \mathbf{u}=\mathbf{0} \in \Omega
$$

можно лишь в том случае, если деформации удовлетворяют уравнению совместности

$$
\frac{\partial^{2} \varepsilon_{11}^{*}}{\partial x_{2}^{2}}+\frac{\partial^{2} \varepsilon_{22}^{*}}{\partial x_{1}^{2}}-\frac{\partial^{2} \varepsilon_{12}^{*}}{\partial x_{1} \partial x_{2}}=0
$$

พับ

$$
[\Re] \varepsilon^{*}=\mathbf{0} \in \Omega,
$$

где

$[\mathscr{B}]=$\begin{tabular}{|c|c|c|}
\hline$\frac{\partial^{2}}{\partial x_{2}^{2}}$ & $\frac{\partial^{2}}{\partial x_{1}^{2}}$ & $-\frac{\partial^{2}}{\partial x_{1} \partial x_{2}}$ \\
\hline
\end{tabular}

Отсюда следует вывод, что минимальный принцип наименьшей работы (принцип Кастильяно) и уравнения совместности деформаций тождественны между собой. Следует отметить, что в теории и расчетах они не могут полностью заменять друг друга. Соблюдение принципа Кастильяно приводит лишь к автоматическому выполнению всех известных уравнений неразрывности (однако число независимых уравнений - особенно это относится к трехмерным задачам - вышеприведенным выводом не регламентируется). (27) является необходимым и достаточным условием интегрирования уравнений $\varepsilon=[\mathscr{A}]^{T} \mathbf{u}$.

Второй способ. Уравнение совместности (27) позволяет получить и следующие зависимости:

$$
[\mathscr{B}][\mathscr{A}]^{T} \mathbf{u}=\mathbf{0} \text { и }[\mathscr{A}][\mathscr{B}]^{T}=\mathbf{0} .
$$

На основе (28) решение $\sigma$ однородной системы уравнений $[\mathscr{A}] \boldsymbol{\sigma}=\mathbf{0}$ можно представить в виде:

$$
\sigma=[\Re]^{T} \varphi \in \Omega,[N][\Re]^{T} \varphi=\mathbf{q} \text { на } \mathscr{L}_{f} .
$$

Здесь $\varphi\left(x_{1}, x_{2}\right)-$ функщия напряжений. Тогда условия Куна-Таккера для задачи (13)-(15) принимают вид:

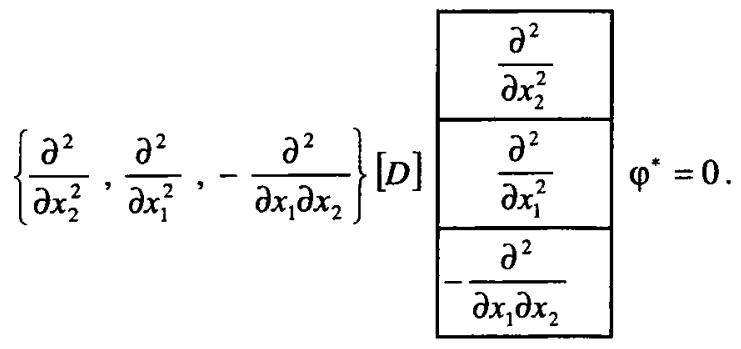

Так огределяется функция $\varphi^{*}$ по бигармоническому уравнению $\nabla^{4} \varphi^{*}=0$ :

$$
\frac{\partial^{4} \varphi^{*}}{\partial x_{1}^{4}}+2 \frac{\partial^{4} \varphi^{*}}{\partial x_{1}^{2} \partial x_{2}^{2}}+\frac{\partial^{4} \varphi^{*}}{\partial x_{2}^{4}}=0
$$

при $\mathbf{u}=\mathbf{0} \in \mathscr{L}_{u}$.

Статические граничные условия $[N][\mathscr{B}]^{T} \varphi^{*}=\mathbf{q}$ удовлетворены, так как $\varphi^{*}$ - оптимальное решение задачи (13)-(15).

Третий способ. Преследуется цель определить уравнения метода сил, то есть уравнения (27) с неизвестными функцией $\varphi$ и одним напряжением при соблюдении уравнения равновесия и заданньх граничных условий. Статически возможный вектор

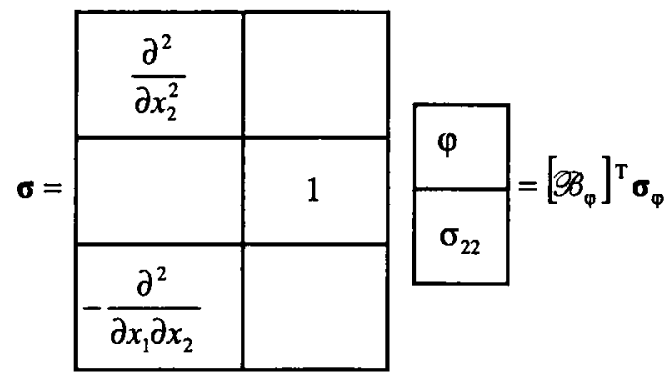

в этом случае регламентируется одним уравнением равновесия (вторым из системы (23))

$$
\frac{\partial^{3} \varphi}{\partial x_{1}^{2} \partial x_{2}}-\frac{\partial \sigma_{22}}{\partial x_{2}}=0 \in \Omega
$$

и граничными условиями, выраженными через функцию $\varphi$ и напряжение $\sigma_{22}$,

$$
[N]\left[\mathscr{B}_{\varphi}\right]^{T} \sigma_{\varphi}=\mathbf{q} \in \mathscr{L}_{f} .
$$

Тогда для задачи анализа (13)-(15) необходимые и достаточные условия оптимальности решения $\sigma_{\varphi}^{*}$ будут следующими:

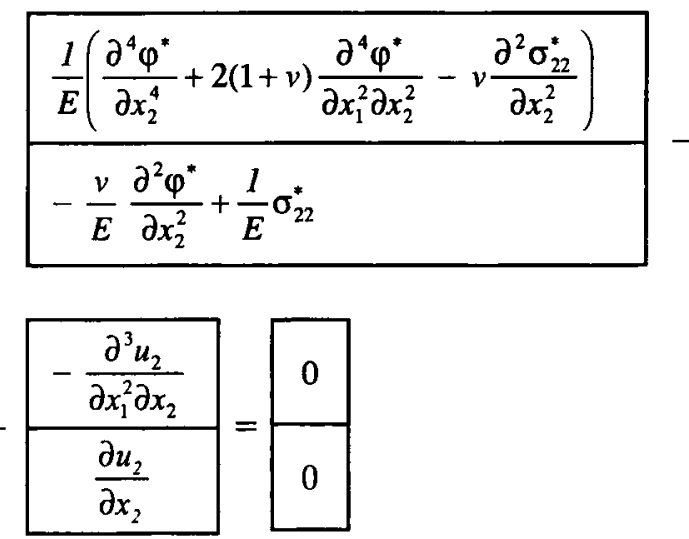

и граничные условия

$$
\mathbf{u}=\mathbf{0} \text { на } \mathscr{L}_{u} .
$$


Второе уравнение по системе (34) информативно. С одной стороны, это геометрическое уравнение

$$
\varepsilon_{22}^{*}=\frac{\partial u_{2}}{\partial x_{2}}
$$

с другой - структура формулы деформации

$$
\varepsilon_{22}^{*}=\frac{l}{E}\left(\sigma_{22}^{*}-v \frac{\partial^{2} \varphi^{*}}{\partial x_{2}^{2}}\right)
$$

косвенно подтверждает существование зависимости

$$
\sigma_{11}^{*}=\frac{\partial^{2} \varphi^{*}}{\partial x_{2}^{2}}
$$

В дальнейшем производится исключение второго уравнения из системы (34). С этой целью оно дважды дифференцируется относительно $x_{1}$ :

$$
\frac{\partial^{3} u_{2}}{\partial x_{1}^{2} \partial x_{2}}=-\frac{\nu}{E} \frac{\partial^{4} \varphi^{*}}{\partial x_{1}^{2} \partial x_{2}^{2}}+\frac{l}{E} \frac{\partial^{2} \sigma_{22}^{*}}{\partial x_{1}^{2}} .
$$

Тогда уравнение-тождество совместности деформаций для плоской области $\Omega$ принимает следуюший вид:

$\frac{\partial^{4} \varphi^{*}}{\partial x_{2}^{4}}+2 \frac{\partial^{4} \varphi^{*}}{\partial x_{1}^{2} \partial x_{2}^{2}}+v \frac{\partial^{4} \varphi^{*}}{\partial x_{1}^{2} \partial x_{2}^{2}}-v \frac{\partial^{2} \sigma_{22}^{*}}{\partial x_{2}^{2}}+\frac{\partial^{2} \sigma_{22}^{*}}{\partial x_{1}^{2}}=0$

при наличии граничных условий

$$
\mathbf{u}=\mathbf{0} \text { на } \mathscr{L}_{u} .
$$

Уравнение метода сил в функциональном пространстве представляется в контексте зависимостей (12):

$$
\begin{aligned}
& \left\{\begin{array}{l}
\frac{\partial^{3} \varphi}{\partial x_{1}^{2} \partial x_{2}}-\frac{\partial \sigma_{22}}{\partial x_{2}}=0, \\
\frac{\partial^{4} \varphi}{\partial x_{2}^{4}}+2 \frac{\partial^{4} \varphi}{\partial x_{1}^{2} \partial x_{2}^{2}}+v \frac{\partial^{4} \varphi}{\partial x_{1}^{2} \partial x_{2}^{2}}- \\
-v \frac{\partial^{2} \sigma_{22}}{\partial x_{2}^{2}}+\frac{\partial^{2} \sigma_{22}}{\partial x_{1}^{2}}=0, \\
{[N]\left[\mathscr{B}_{\varphi}\right] \sigma_{\varphi}=\mathbf{q} \in \mathscr{L}_{f},} \\
\mathbf{u}=\mathbf{0} \in \mathscr{L}_{\mathbf{u}},
\end{array}\right\} \in \Omega, \\
& \sigma_{\varphi}=\left\{\varphi, \sigma_{22}\right\}^{T}, \quad \mathbf{q}=\left\{\mathrm{q}_{1}, \mathrm{q}_{2}\right\}^{T}, \mathbf{u}=\left\{\mathrm{u}_{1}, \mathrm{u}_{2}\right\}^{T} .
\end{aligned}
$$

Таким образом, уравнения в силовых факторах (37) представлены в корректной форме: при заданных граничных условиях для определения искомых функций $\varphi$ и $\sigma_{22}$ в плоской области $\Omega$ имеются и два уравнения. Так как в граничные условия $\lfloor N\rfloor\left\lfloor\mathscr{B}_{\varphi}\right\rfloor \boldsymbol{\sigma}_{\varphi}=\mathbf{q} \in \mathscr{L}_{f}$ входят как $\varphi$, так и $\sigma_{22}$, то система (37) не отвечает традиционной форме уравнений метода сил строительной механики. Система (37) при использовании уравнения равновесия

$$
\frac{\partial^{3} \varphi}{\partial x_{1}^{2} \partial x_{2}}-\frac{\partial \sigma_{22}}{\partial x_{2}}=0
$$

сводится к виду

$$
\left\{\begin{array}{l}
\nabla^{4} \varphi=0 \in \Omega, \\
{\left[N_{\varphi}\right] \varphi=\mathbf{q} \in \mathscr{L}_{f},} \\
\mathbf{u}=\mathbf{0} \in \mathscr{L}_{u} .
\end{array}\right.
$$

Здесь

$$
\left[N_{\varphi}\right]=\left[\begin{array}{c}
n_{1} \frac{\partial^{2}}{\partial x_{2}^{2}}-n_{2} \frac{\partial^{2}}{\partial x_{1} \partial x_{2}} \\
-n_{1} \frac{\partial^{2}}{\partial x_{1} \partial x_{2}}+n_{2} \frac{\partial^{2}}{\partial x_{1}^{2}}
\end{array} .\right.
$$

\section{5. Условия Куна-Таккера для трехмерной задачи}

Статически возможный вектор напряжений для задачи анализа (13)-(15) может быть определен по формуле:

$$
\sigma=[\Re]^{T} \varphi .
$$

Здесь

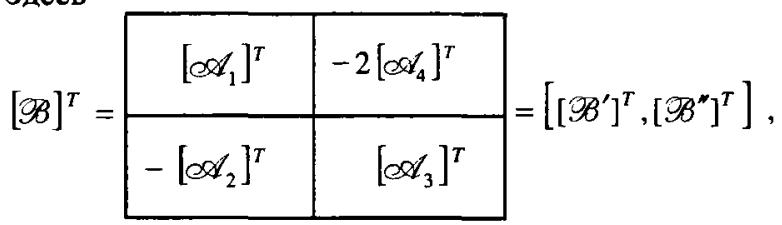

$\varphi=\left\{\varphi_{1}, \varphi_{2}\right\}^{T}=\left\{\varphi_{1}, \varphi_{2}, \varphi_{3}, \varphi_{4}, \varphi_{5}, \varphi_{6}\right\}^{T}-$ вектор функции напряжений. Матрица $[\mathscr{B}]^{T}$ приведена в приложении. Уравнения равновесия (14)

$$
-[\mathscr{A}][\mathscr{B}]^{T} \varphi=0
$$

в этом случае удовлетворяются тождественно. Тогда условия Куна-Таккера превращаются в

$$
\nabla \mathscr{F}\left(\mathbf{x}^{*}\right)=\mathbf{0}
$$

и для оптимального решения $\varphi^{*}$ задачи (13)-(15) принимают вид: 


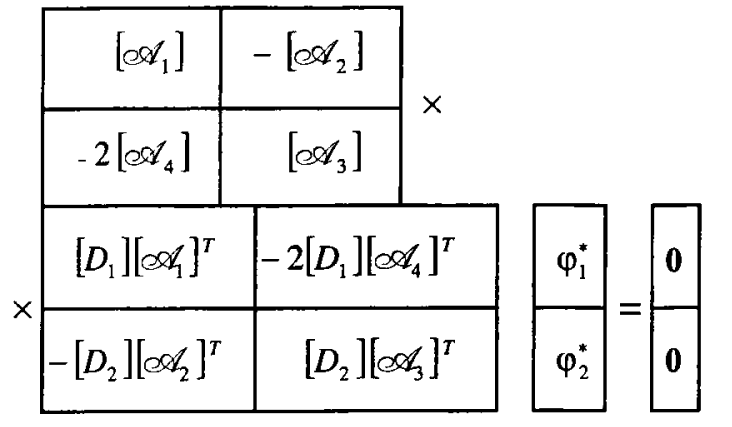

при условиях $\mathbf{u}=\mathbf{0} \in A_{u}$.

Уравнения (40) являются уравнениями совместности деформаций для упругого тела (10): как указывалось выше, минимальный принцип дополнительной энергии и уравнения неразрывности тождественны между собой.

Система уравнений теории упругости

$$
[\mathscr{B}][D][\mathscr{B}]^{T} \varphi=\mathbf{0} \in V
$$

с шестью неизвестными функциями напряжений $\varphi$ решается при учете краевых условий

$$
\begin{gathered}
{[N][\mathscr{B}]^{T} \varphi=\mathbf{q} \in A_{f},} \\
\mathbf{u}=\mathbf{0} \in A_{u} .
\end{gathered}
$$

Таким образом, задача теории упругости в силовых переменных (41)-(43) сформулирована корректно: вместо задачи определения шести неизвестных функций напряжений $\sigma$ по системе девяти уравнений (11) здесь требуется определить шесть неизвестных функций напряжений $\varphi$ по системе шести уравнений при заданных граничных условиях.

Удовлетворяюшие уравнениям равновесия в объеме тела $V$ векторы напряжений для трехмерной

\begin{tabular}{|c|c|c|}
\hline$\frac{\partial^{2}}{\partial x_{2}^{2}}$ & $\frac{\partial^{2}}{\partial x_{3}^{2}}$ & \\
\hline \multirow{4}{*}{$\partial x_{1}^{2}$} & & $\partial^{2}$ \\
\hline & & $\overline{\partial x_{3}^{2}}$ \\
\hline & $\partial^{2}$ & $\partial^{2}$ \\
\hline & $\overline{\partial x_{1}^{2}}$ & $\overline{\partial x_{2}^{2}}$ \\
\hline \multirow{4}{*}{$-\frac{\partial^{2}}{\partial x_{1} \partial x_{3}}$} & & \\
\hline & & \\
\hline & $-\frac{\partial^{2}}{\partial x_{1} \partial x_{3}}$ & \\
\hline & & $-\frac{\partial^{2}}{\partial x_{2} \partial x_{3}}$ \\
\hline
\end{tabular}
задачи теории упругости (13)-(15) формально могут быть подобраны и так:

или

$$
\begin{array}{|c|c|c|}
\hline-2 \frac{\partial^{2}}{\partial x_{2} \partial x_{3}} & & \\
\hline & -2 \frac{\partial^{2}}{\partial x_{1} \partial x_{3}} & \\
\hline & & -2 \frac{\partial^{2}}{\partial x_{1} \partial x_{2}} \\
\hline \frac{\partial^{2}}{\partial x_{1} \partial x_{3}} & \frac{\partial^{2}}{\partial x_{2} \partial x_{3}} & -\frac{\partial^{2}}{\partial x_{3}^{2}} \\
\hline \frac{\partial^{2}}{\partial x_{1} \partial x_{2}} & -\frac{\partial^{2}}{\partial x_{2}^{2}} & \frac{\partial^{2}}{\partial x_{2} \partial x_{3}} \\
\hline-\frac{\partial^{2}}{\partial x_{1}^{2}} & \frac{\partial^{2}}{\partial x_{1} \partial x_{2}} & \frac{\partial^{2}}{\partial x_{1} \partial x_{3}} \\
\hline \varphi_{4}
\end{array}
$$

Использование вектора $\sigma^{\prime}$ приводит к первым трем уравнениям совместности деформаций, то есть $к$ системе (11 б) в виде:

$$
[\mathscr{O A} 1]\left[D_{1}\right] \boldsymbol{\sigma}_{1}^{\prime}-[\mathscr{O A} 2]\left[D_{2}\right] \boldsymbol{\sigma}_{2}^{\prime \prime}=\mathbf{0} \in V,
$$

а использование вектора $\sigma^{\prime \prime}-$ к уравнениям (11 в) в виде:

$$
-2\left[\mathscr{A}_{4}\right]\left[D_{1}\right] \sigma_{1}^{\prime \prime}+\left[e \mathscr{A}_{3}\right]\left[D_{2}\right] \boldsymbol{\sigma}_{2}^{\prime \prime}=\mathbf{0}
$$

В этих двух случаях краевые условия на $A_{f}$ различны между собой и отличны от условий (42). Статически возможный вектор можно получить и перестановкой (заменой) столбцов в выражениях (44) и (45). Так как краевые условия (42)-(43) не вызывают сомнений, то следует вывод, что задачи (46) и (47) сформулированы некорректно. С целью соблюдения условий совместности деформаций на поверхности тела $A$ (как в задаче (41)-(43)) решение задачи анализа $\sigma$ в дальнейшем формально представляется как сумма напряжений $\rho$ и $\sigma_{e}$, т.е. $\sigma=\rho+\sigma_{e}$. Пусть $\sigma_{e}-$ некоторый известный статически возможный вектор, на который распространяются требования

$$
\begin{gathered}
-[\varrho f] \sigma_{e}=0 \in V, \\
{[N]\left(\rho+\sigma_{e}\right)=\mathbf{q} \in A_{f} .}
\end{gathered}
$$

Здесь $\rho$ - искомое самоуравновешенное в объеме тела $V$ напряженное состояние, т.е.

$$
-[\infty /] \rho=0 \in V .
$$

Тогда задача анализа (13)-(15) может быть представлена так:

найти 


$$
\min \frac{1}{2} \int\left(\rho+\sigma_{e}\right)^{T}[D]\left(\rho+\sigma_{e}\right) d V
$$

при условиях

$$
\begin{gathered}
\mathscr{A} \boldsymbol{\rho}=\mathbf{0} \in V, \\
N\left(\boldsymbol{\rho}+\boldsymbol{\sigma}_{e}\right)=\mathbf{q} \in A_{f} .
\end{gathered}
$$

Пусть напряжение $\sigma_{e}$ определяется согласно зависимости (45) $\sigma_{e}=\left[\mathscr{B}^{\prime \prime}\right]^{T} \varphi_{2}$, а напряжение $\rho$ выражается через функции напряжения $\varphi_{1}$. Тогда условия Куна-Таккера для оптимального решения задачи (50)-(52) $\rho^{*}$ принимают вид:

$\left[\mathscr{B}^{\prime}\right][D]\left[\mathscr{B}^{\prime}\right]^{T} \varphi_{1}^{*}+\left[\mathscr{B}^{\prime}\right][D]\left[\mathscr{B}^{\prime \prime}\right]^{T} \varphi_{2}^{*}=\mathbf{0} \in V$,

$\mathbf{u}=\mathbf{0} \in A_{u}$.

Полная система уравнений теории упругости (7) такова:

$$
\left\{\begin{array}{l}
{\left[\mathscr{B}^{\prime}\right][D]\left[\mathscr{B}^{\prime}\right]^{T} \varphi_{1}+\left[\mathscr{B}^{\prime}\right][D]\left[\mathscr{B}^{\prime \prime}\right]^{T} \varphi_{2}=\mathbf{0} \in V} \\
{[N]\left(\left[\mathscr{B}^{\prime}\right]^{T} \varphi_{1}+\left[\mathscr{B}^{\prime \prime}\right]^{T} \varphi_{2}\right)=\mathbf{q} \in A_{f}} \\
\mathbf{u}=\mathbf{0} \in A_{u}
\end{array}\right.
$$

Первая группа уравнений по системе (54) - это уравнения совместности в форме Бельтрами (11 б). Система (54) отвечает доказанному Васидзу принципу относительно уравнений совместности деформаций Сен-Венана [3]:

если условия совместности деформаций удовлетворены на поверхности исследуемого тела А, то для интегрируемости уравнений
$\varepsilon-[\propto]^{T} \mathbf{u}=\mathbf{0}$ необходимо $и$ достаточно удовлетворения зависимостей либо первой группы (10 а), либо только второй группы (10 б) из системы уравнений Сен-Венана.

\section{6. Выводы}

Уравнения совместности деформаций СенВенана для идеально упругого тела рассматриваются с привлечением теории математического программирования. С этой целью на основе принципа минимума дополнительной энергии построена экстремальная задача анализа для статически возможных напряжений. Показано, что уравнения совместности деформаций есть известные в теории математического программирования условия оптимальности КунаТаккера для решаемой задачи анализа.

Выявлена возможность получения различных формулировок уравнений совместности в зависимости от формы представления статически возможных векторов напряжений. Для трехмерной задачи доказана возможность получения шести уравнений теории упругости в напряжениях (с привлечением вектор-функции напряжений с шестью компонентами). До сих пор решение задачи теории упругости в напряжениях обычно связывалось с определением шести компонентов вектора напряжений из девяти уравнений равновесия и Бельтрами.

Применение условий Куна-Таккера подтверждает оригинальный и пока мало распространенный вывод Васидзу о необходимых и достаточных условиях интегрируемости геометрических уравнений Коши.

Приложение. Сопряженный дифференциальный оператор уравнений совместности деформаций

$[\because]^{T}=$\begin{tabular}{|c|c|c|c|c|c|}
\hline$\frac{\partial^{2}}{\partial x_{2}^{2}}$ & $\frac{\partial^{2}}{\partial x_{3}^{2}}$ & & $-2 \frac{\partial^{2}}{\partial x_{2} \partial x_{3}}$ & & \\
\hline$\frac{\partial^{2}}{\partial x_{1}^{2}}$ & & $\frac{\partial^{2}}{\partial x_{3}^{2}}$ & & $-2 \frac{\partial^{2}}{\partial x_{1} \partial x_{3}}$ & \\
\hline & $\frac{\partial^{2}}{\partial x_{1}^{2}}$ & $\frac{\partial^{2}}{\partial x_{2}^{2}}$ & & & $-2 \frac{\partial^{2}}{\partial x_{1} \partial x_{2}}$ \\
\hline$-\frac{\partial^{2}}{\partial x_{1} \partial x_{2}}$ & & & $\frac{\partial^{2}}{\partial x_{1} \partial x_{3}}$ & $\frac{\partial^{2}}{\partial x_{2} \partial x_{3}}$ & $-\frac{\partial^{2}}{\partial x_{3}^{2}}$ \\
\hline & $-\frac{\partial^{2}}{\partial x_{1} \partial x_{3}}$ & & $\frac{\partial^{2}}{\partial x_{1} \partial x_{2}}$ & $-\frac{\partial^{2}}{\partial x_{2}^{2}}$ & $\frac{\partial^{2}}{\partial x_{2} \partial x_{3}}$ \\
\hline & & $-\frac{\partial^{2}}{\partial x_{2} \partial x_{3}}$ & $-\frac{\partial^{2}}{\partial x_{1}^{2}}$ & $\frac{\partial^{2}}{\partial x_{1} \partial x_{2}}$ & $\frac{\partial^{2}}{\partial x_{1} \partial x_{3}}$ \\
\hline
\end{tabular}




\section{Литература}

1. Л. А. Розин. Вариационные постановки задач для упругих систем. Л.: Изд-во Ленингр. ун-та, 1978. 224 с.

2. К. Ф. Черных, Ю. 3. Алешков, В. В. Понятовский, В. А. Шамина. Введение в механику сплошных сред. Л.: Изд-во Ленингр. ун-та, 1984. 280 с.

3. К. Васидзу. Вариационные методы в теории упругости и пластичности. Москва: Мир, 1987. 542 с.

4. R. V. Soothwell. Castigliano's principle of minimum strain energy // Proceedings of the Royal Society, 1936, v. 154. N. 881, p. 4-21.

5. J. Grycz. On the compatibility conditions in the classica theory of elasticity // Archivium mechaniki stosowaney, Nr 6, 19, Warszawa: Panstawowe wydawnictwo naukowe, 1967, p. 883-891.

6. В. Новацкий. Теория упругости. М.: Мир, 1975. 872 с.

7. В. Д. Клюшников. Вывод уравнений БельтрамиМичелла из вариационного уравнения Кастильяно // ПММ, том XVIII, вып. 2, 1954, с. 722-731.

8. Б. Ф. Власов. О числе независимых уравнений неразрывности // Труды ун-та дружбы народов им. Патриса Лумумбы, том XXXIV, вып. 5, М., 1968. с. 171-174.

9. Cyras. Mathematical models for the analysis and optimization of elastoplastic structures. New York: John Wiley, 1983. $121 \mathrm{p}$.

10. Ю. Ю. Аткочюнас. Условия Куна-Таккера в задачах приспособляемости // Statyba (Строительство), № 1(5), Вильнюс: Техника, 1996, с. 14-28.

11. М. Базара, К. Шетти. Нелинейное программирование М.: Мир, 1982. 583 с.

Iteikta 20000223

\section{KUNO IR TAKERIO SAZYGOS SUDARANT TAMPRU- MO TEORIJOS LYGTIS ITEMPIMAIS}

\section{E. Chraptovix, J. Atkočiûnas}

\section{S a n t r a u k a}

Tamprumo teorijos lygčiụ jtempimais sprendimas siejamas su radimu šešiu ittempimų vektoriaus komponentu, tenkinančiu tris kūno pusiausvyros lygtis ir šešias Beltrami priklausomybes bei kraštines salygas. Tiesioginis devynių diferencialiniụ dalinèmis išvestinemis lygčių sistemos integravimas šešių ieškomų itempimų funkciju atžvilgiu yra sunkus. Todèl dažnai kraštinis tamprumo teorijos uždavinys itempimais sprendžiamas variaciniu Kastiljano principu, leidžiančiu gauti tapatinga šešiu Sen-Venano deformaciju darnos lygčiu tenkinimą (Sausvelo, Kliušnikovo ir kitu darbai). Tačiau tiesiogiai šis principas nereglamentuoja nepriklausomu deformaciju darnos lygčių skaičiaus. Lieka neaišku, ar tamprumo teorijos lygčių jtempimais sistema nèra perteklinè.

Šiame straipsnyje Sen-Venano deformacijų darnos lygtys idealiai tampriam kũnui nagrinèjamos matematinio programavimo teorijos kontekste. Tam energinio principo apie papildomos energijos minimuma pagrindu sudarytas ekstremumo analizès uždavinio statiškai galimiems įtempimams modelis. Parodyta, jog deformaciju darnos lygtys yra pagal matematinio programavimo teoriją sprendžiamo analizès uždavinio Kuno ir Takerio optimalumo sąlygos. Straipsnyje pasinaudota galimybe ivairiai formuoti deformacijų darnos lygtis priklausomai nuo statiškai galimus itempimus apibrěžiančiu sąlygu. Pagal jtempimu funkcijos su sešais komponentais vektoriu irodyta galimybe gauti šešias trimačio tamprumo teorijos uždavinio deformacijų damos lygtis.

Straipsnyje Kuno ir Takerio salygomis patvirtinta originali ir kol kas ne itin paplitusi Vasidzu išvada apie būtinas ir pakankamas geometrinių Koši lygčių integruojamumo sąlygas.

\section{ROLE OF KUHN-TUCKER CONDITIONS IN ELASTI- CITY EQUATIONS IN TERMS OF STRESSES}

\section{E. Chraptovič, J. Atkociūnas}

\section{S u m mary}

Solution of the elasticity problem in terms of stresses leads to the stress vector six components, satisfying the Beltram compatibility eqns and boundary conditions, evaluation. A direct integration of the nine differential eqns system in respect of the six stress components is difficult to realise practically. This is the reason why often the Castigliano variation principle to solve the boundary elasticity problem in terms of stresses is applied. An application of the above-mentioned principle ensures the satisfaction of all the six Saint-Venant strain compatibility eqns (see the works of Southwell, Kliushnikov, a.o.). Castigliano variation principle does not define the number of independent strain compatibility eqns. Thus, it is not clear whether the elasticity problem eqns system in terms of stresses is overdefined or not

The strain compatibility eqns for an ideal elastic body is investigated in the article by means of the mathematical programming theory. A mathematical model to evaluate the statically admissible stresses is formulated on the basis of complementary energy minimum principle. It is proved that the strain compatibility eqns mean the Kuhn-Tucker optimality conditions of the mathematical programming problem. The method to formulate the strain compatibility eqns in respect of the statically admissible stresses defining eqns formulation technique is revealed. The proposed method is illustrated to achieve the six component stresses vector in functional space for the threedimension problem: usually the solution of the elasticity problem in terms of the stresses is realised via the nine eqns system integration.

The Kuhn-Tucker conditions allowed to confirm an original but not usually applied Washizu conclusion about Cauchy geometrical compatibility eqns.

Ela CHRAPTOVIČ. Doctoral student. Dept of Structural Mechanics, Vilnius Gediminas Technical University. Sauletekio al. 11, LT-2040 Vilnius, Lithuania.

E-mail: Ela.Chraptovic@st.vtu.It

Civil engineer (1995). MSc (civil engineering, 1997). Research interests: structural mechanics, optimisation of elastoplastic structures.

Juozas ATKOČIŪNAS. Doctor Habil, Professor. Head of the Dept of Structural Mechanics, Vilnius Gediminas Technical University. Sauletekio al. 11, LT-2040 Vilnius, Lithuania. E-mail: Juozas.Atkociunas@ @st.vtu.lt

Civil engineer (1967). Dr Eng (structural mechanics, 1973). Dr Habil (mechanics, 1996). Research interests: structural and computational mechanics, applied mathematical programming, analysis and optimisation of dissipative structures under repeated-variable loading. 